\title{
Recovery of Pseudomonas aeruginosa in respiratory specimens from HIV positive patients being evaluated for Pneumocystis carinii pneumonia
}

\author{
Ramona L Doyle, Joseph J Doherty, Leslie H Zimmerman
}

\begin{abstract}
Background - Despite the immune suppression, frequent hospital admissions, and many intercurrent illnesses associated with HIV infection, Pseudomonas aeruginosa has been cited relatively infrequently as a respiratory pathogen in HIV positive patients.

Methods - The microbiological isolates, medical records, radiographic reports, and laboratory data from 224 patients undergoing sputum induction and/or bronchoalveolar lavage for evaluation of respiratory symptoms suspicious for Pneumocystis carinii pneumonia (PCP) from 1989 to 1992 were reviewed retrospectively.

Results - An increasing number of respiratory isolates with Pseudomonas aeruginosa was found over this time period. Eighteen of the 224 patients were identified in whom $P$ aeruginosa was recovered on at least one occasion. These patients were more likely to have a history of smoking and prior PCP than those in whom Pseudomonas was not recovered. Mean CD4 counts were also significantly lower in these patients.

Conclusions - Pseudomonas aeruginosa may be recovered from a substantial number of respiratory isolates from HIV positive patients suspected of having PCP. The prevalence of this phenomenon may be increasing.

(Thorax 1995;50:548-550)
\end{abstract}

Cardiovascular

Research Institute,

University of

California at San

Francisco

R L Doyle

Division of Pulmonary and Critical Care

Medicine, San

Francisco Veterans

Administration

Medical Center,

San Francisco,

California 94121,

USA

J J Doherty

L H Zimmerman

Reprint requests to: Dr L H Zimmerman.

Received 9 November 1994

Accepted for publication

19 January 1995
Keywords: Pseudomonas aeruginosa, HIV, Pneumocystis carinii pneumonia.

Pseudomonas aeruginosa is a respiratory pathogen found most commonly in patients admitted to hospital, those undergoing prolonged mechanical ventilation, patients with neutropenia or on immunosuppressive drugs, and those with cystic fibrosis. Despite the immune suppression, frequent hospital admissions, and many intercurrent illnesses associated with HIV infection, Pseudomonas has been cited relatively infrequently as a respiratory pathogen in HIV positive patients. ${ }^{1-3}$ While early studies of Pseudomonas in HIV positive patients reported predominantly non-pulmonary nosocomial disease, ${ }^{45}$ a recent study from a large outpatient AIDS clinic reported 14 cases of community- acquired pulmonary disease due to $P$ aeruginosa. $^{6}$

Recent evidence suggests that improved $\stackrel{2}{\overrightarrow{2}}$ medical care, earlier intervention, and im- ov proved treatment has led to improved survival o among HIV positive patients. ${ }^{78}$ As patients with of HIV live longer the clinical manifestations of $\infty$

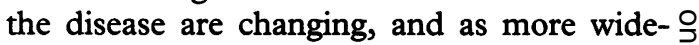
spread prophylaxis for Pneumocystis carinii, $\overrightarrow{-}$ Mycobacterium tuberculosis, and Mycobacterium avium becomes the standard of care, an increasing incidence of other respiratory patho- $\vec{\theta}$ gens such as $P$ aeruginosa may occur. It is our $v$ current clinical practice to evaluate all HIV positive patients with suspected Pneumocystis carinii pneumonia (PCP) with sputum induction followed by bronchoalveolar lavage if sputum is non-diagnostic. We retrospectively $\stackrel{\mathbb{2}}{2}$ reviewed bacterial isolates from all $\mathrm{HIV}$ positive $\overrightarrow{\overrightarrow{0}}$ patients undergoing sputum induction and/or 3 bronchoalveolar lavage for suspected PCP from 1989 to 1992 and noted an increasing frequency of $P$ aeruginosa recovery during this $\vec{\partial}$ time. We describe this group of patients, their clinical course, and compare them with all ${ }_{0}^{\times}$ patients undergoing similar evaluation for PCP 3 . during this period.

\section{Methods}

We retrospectively reviewed the micro- 글 biological isolates, medical records, radio- N graphic reports, clinical history, and laboratory $N$ data of all HIV positive patients suspected of having the diagnosis of PCP who underwent ${ }_{\sigma}^{\omega}$ sputum induction and/or bronchoalveolar lavage from 1989 to 1992 . At San Francisco Veteran's Administration Medical Center all $\stackrel{\oplus}{\oplus}$ sputum induction and bronchoalveolar lavage 0 specimens from HIV positive patients are $\stackrel{\vec{P}}{\mathrm{P}}$ routinely submitted for bacterial, fungal, and $\stackrel{\Phi}{\Phi}$ mycobacterial culture.

A subgroup of patients was identified with respiratory isolates positive for $P$ aeruginosa on 0 at least one occasion during this time period;응 this group is identified as the "+ PsA" group. The clinical, radiographic, and laboratory findings of this group were compared with those patients in whom Pseudomonas was not recovered ("-PsA" group). In the + PsA patients we also reviewed antiretroviral therapy, PCP prophylaxis, and subsequent clinical course.

Patients were considered to be smokers if they were currently smoking or, despite having quit, had a significant smoking history $(>10$ 
Table 1 Comparison of clinical characteristics of HIV positive patients undergoing bronchoalveolar lavage or sputum induction in whom Pseudomonas aeruginosa was $\left(+P_{s} A\right)$ or was not $(-P s A)$ recovered $(n=224)$

\begin{tabular}{lll}
\hline & $-P s A$ & $+P s A$ \\
& $(n=206)$ & $(n=18)$ \\
\hline Sex (male) & 206 & 17 \\
Smoking history & $82(40 \%)$ & $13(72 \%)^{*}$ \\
Prior PCP & $58(28 \%)$ & $10(56 \%)^{*}$
\end{tabular}

$\mathrm{PCP}=$ Pneumocystis carinii pneumonia

${ }^{*} \mathrm{p}<0.05$ (Student's $t$ test).

Table 2 Comparison of CD4 counts and recovery of Pneumocystis carinii from 324 respiratory episodes between 1989 and 1992

\begin{tabular}{lcl}
\hline & $\begin{array}{l}-P S A \\
(n=302)\end{array}$ & $\begin{array}{l}+P s A \\
(n=22)\end{array}$ \\
\hline $\begin{array}{l}\text { Recovery of PsA } \\
\text { PsA sole pathogen }\end{array}$ & 0 & 22 \\
$\begin{array}{l}\text { Recovery of } \\
\text { Pneumocystis carinii }\end{array}$ & $152(50 \%)$ & $8(45 \%)$ \\
$\begin{array}{l}\text { Mean (SD) CD4 } \\
\left.\text { count (mm }{ }^{3}\right)\end{array}$ & $66.5(87 \cdot 7)$ & $18 \cdot 4(25 \cdot 4)^{*}$ \\
\hline
\end{tabular}

PsA $=$ Pseudomonas aeruginosa

$* \mathrm{p}<0.025$.

Table 3 Comparison of chest radiographic patterns for episodes of respiratory complaints

\begin{tabular}{lll}
\hline Radiographic patterns & $(n=302)$ & $+P_{S} A$ \\
\hline Diffuse infiltrate & $233(77 \%)$ & $14(64 \%)$ \\
Focal abnormality & $52(17 \%)$ & $6(27 \%)$ \\
Normal & $15(5 \%)$ & $1(4 \%)$ \\
Lost & 2 & \\
\hline
\end{tabular}

pack years) and had abstained for less than two years. Radiographs were classified as "focal", "diffuse", or "normal" based on the final radiographic reading by staff radiologists. Radiographic findings categorised as "focal" included focal infiltrates, effusions, masses, and cavities.

CD4 counts were recorded at the time of presentation for each respiratory symptom episode when available, and otherwise were considered for analysis only if they had been

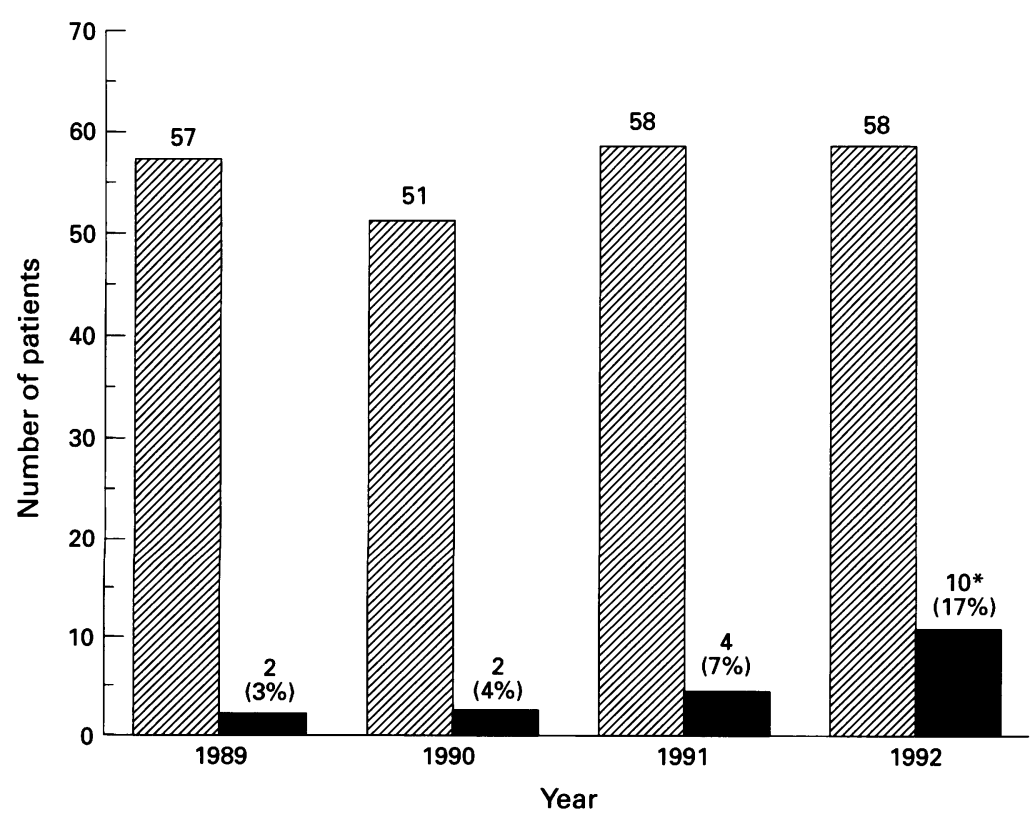

Total number of patients evaluated per year compared with number of patients in whom Pseudomonas aeruginosa was recovered. ${ }^{*} p<0.05$ compared with 1989 ( $\chi^{2}$ test). obtained within four months before the episode. Calculation of the mean $\mathrm{CD} 4$ counts for the +PsA group included values which corresponded only to those episodes in which $P$ aeruginos $a$ was actually recovered - that is, if a patient had four evaluations for symptoms during this period but only twice had recovery of $P$ aeruginosa, only those CD4 counts corresponding to the episodes in which Pseudomonas was recovered were used.

Cultures from bronchoscopes used for bronchoalveolar lavage are routinely obtained to rule out nosocomial contamination and were negative for Pseudomonas or any other bacteria during the time of this analysis.

\section{DATA ANALYSIS}

Data are expressed as mean (SD). Statistical analysis was performed using the Student's $t$ test. Chi square analysis was used to compare the proportions in the figure, a $p$ value of $<0.05$ being considered significant.

\section{Results}

Between 1989 and 1992224 HIV positive patients presented with 324 episodes of respiratory complaints prompting an evaluation (sputum induction and/or bronchoalveolar lavage) for PCP. Eighteen patients were identified in whom $P$ aeruginosa was recovered on at least one evaluation. The clinical characteristics of the - PsA group $(n=206)$ and the +PsA group $(n=18)$ are described in table 1 . In the + PsA group patients were more likely to be current smokers and to have had prior episodes of PCP than the -PsA group.

The total number of episodes of respiratory complaints prompting sputum induction and/ or bronchoalveolar lavage in the two patient groups is compared in table 2. Among the 18 patients from whom $P$ aeruginosa was recovered at least once, there were 32 episodes prompting either sputum induction and/or bronchoalveolar lavage for suspected PCP. $P$ aeruginosa was recovered in 22 of these 32 episodes, and was the only pathogen recovered in 14 of the episodes. Eight patients had more than one respiratory episode during this time period; one patient had four episodes. Once $P$ aeruginosa had been recovered in a respiratory specimen any subsequent sputum induction and/or bronchoalveolar lavage specimens were found to be positive for this organism. Recovery of Pneumocystis carinii in the two groups was similar.

Chest radiographs were available for 322 episodes of respiratory symptoms (table 3 ). All patterns were seen in patients from whom $P$ aeruginosa was recovered and there was no significant difference in findings between the two groups. Focal findings consisted of upper lobe cavities in three patients, upper lobe infiltrates in two patients, and an upper lobe nodule in one patient.

Seventeen of the 18 patients with recovery of Pseudomonas in either bronchoalveolar lavage or sputum induction specimens were inpatients at the time of first recovery of the organism; however, most $(16 / 18)$ had short hospital stays 
(less than five days) prior to the recovery of Pseudomonas. In general, the Pseudomonas organisms recovered from these patients were sensitive to first line antipseudomonal therapy (e.g. piperacillin, gentamicin). Only three of the 18 patients received antipseudomonal therapy as defined by 10-14 days of an antipseudomonal antibiotic. Two of the 18 patients were on multiple antibiotics for documented Mycobacterium avium infection and 14 were receiving PCP prophylaxis.

Detailed follow up was available for 16 of the 18 patients in the +PsA group and for 184 of the 206 patients in the -PsA group. The mean (SD) time to death for the 16 patients in the +PsA group was $6 \cdot 0(7 \cdot 5)$ months compared with $11.9(11.5)$ months in the -PsA group, a difference which was significant $(\mathrm{p}<0.05)$.

The data presented in the figure show that from 1989 to 1992 there was an increase in the number of patients from whom $P$ aeruginosa was recovered.

\section{Discussion}

Pseudomonas aeruginosa may be recovered from a substantial number of respiratory isolates from HIV positive patients suspected of having PCP and the prevalence of this phenomenon may be increasing. While Pneumocystis carinii remains a leading cause of pneumonia among HIV positive patients, its diagnosis remains a challenge due to the wide variety of infectious as well as non-infectious processes that may mimic it.

The low CD4 counts in the + PsA patients are likely to reflect the late stage of their HIV disease. The association of $P$ aeruginosa with late HIV infection may represent a propensity for colonisation by Pseudomonas of lungs previously damaged by HIV-related opportunistic infections, or by HIV itself, or both. Indeed, patients in our study in whom Pseudomonas was recovered were twice as likely to have had a prior bout of PCP, and nearly twice as likely to have a history of smoking compared with those in whom Pseudomonas was not recovered. These findings in HIV positive patients may parallel the course of $P$ aeruginosa infection that occurs in patients with lung damage from cystic fibrosis. ${ }^{9}$ Other factors which may increase the risk of Pseudomonas infection in HIV patients include a high incidence of acute and chronic sinusitis, ${ }^{1011}$ frequent use of antibiotics, frequent admissions to hospital, impaired B cell activity, ${ }^{12}$ and impaired neutrophil bactericidal activity. ${ }^{13}$

Previous studies of pseudomonal infection in HIV positive patients have largely been limited to consideration of non-pulmonary infection. The exception to this, a recent retrospective study by Baron and Hollander, ${ }^{6}$ described mostly outpatients with an indolent, subacute course who "recovered" after antibiotic therapy. In their study most patients received adequate antipseudomonal antibiotics but suffered a high rate of relapse. In our study we were able not only to characterise patients in whom Pseudomonas was recovered, but to compare them with other HIV positive patients with similar respiratory complaints. Those few patients who did receive appropriate antibiotics shared the uniformly poor clinical outcome. Contrary to the overall low mortality of pseudo- 음 monal pulmonary disease cited by Baron and $\cong$ Hollander, 10 of the 16 patients in our study died within six months of recovery of the $\stackrel{\oplus}{+}$ organism from a respiratory specimen. This in- $\frac{}{2}$ dicates either the pathogenicity of $P$ aeruginos $a, \frac{\bar{\sigma}}{\bar{c}}$ or the propensity of the organism to colonise $\frac{\pi}{\Phi}$ the respiratory tracts of patients with late stage HIV disease, or both.

The recovery of $P$ aeruginosa from respiratory $\vec{\circ}$ specimens from symptomatic HIV positive $\vec{\overrightarrow{ }}$ patients increased from $3 \%$ in 1989 to $17 \%$ in $\omega$ 1992. The clinical presentation of these $\overrightarrow{\vec{x}}$ patients was typified by fever, dyspnoea, and $\dot{x}$ radiographic evidence of pulmonary infiltrates $O$ - the classic presentation of patients with PCP. In 14 of the 22 episodes, however, $P$ aeruginos $a$ was the only pathogenic organism recovered. 은 These results emphasise the need for definitive $\vec{r}$ diagnosis of pulmonary infiltrates in HIV positive patients. However, a case-control study is needed to determine the risk factors for $\vec{\theta}$ acquisition of $P$ aeruginosa in this patient popu- $P$ lation. Such a study would answer more definitively some of the questions raised by our observations, specifically the role of en- $\bar{\partial}$ vironmental exposures, prophylactic antibiotics, comorbid diseases, and number of $\stackrel{\mathbb{}}{\varrho}$ hospital admissions in the acquisition of Pseudo- $\overrightarrow{\overrightarrow{0}}$ monas, and its overall impact on the morbidity and mortality of HIV positive patients.

1 Kielhofner M, Atmas RL, Hamill RH, Musher DM. Life g threatening Pseudomonas aeruginosa infections in patients with human immunodeficiency virus infection. Clin Infect $\frac{\sigma}{3}$ Dis 1992;14:403-11.

2 Witt DS, Craven DE, McCabe WT. Bacterial infection 2 in adult patients with the acquired immune deficiency $\mathrm{O}$ syndrome (AIDS) and AIDS related complex. Am $\mathcal{f} M e d$ syndrome (AIDS) and AIDS related complex. Am $\mathcal{f}$ Med
1987;82:900-6.

3 Whimby E, Gold JWM, Polsky B, Drianski J, Hawkins C, 근 Brevias A, et al. Bacteremia and fungemia in patients with $D$ the acquired immune deficiency syndrome. Ann Intern 을 Med 1986;104:511-4.

4 Tucker KJ, Anton B, Tucker HJ. The effect of human $\mathrm{N}$ immunodeficiency virus infection on the distribution and outcome of pneumonia in intensive care units. West $\mathcal{F}$ Med $\mathrm{N}$ 1992;157:637-40.

5 Nelson MR, Shanson DC, Barter GJ, Hawkins DA, Gazzard BG. Pseudomonas septicaemia associated with HIV. $₹$ AIDS 1991;5:761-3.

6 Baron AD, Hollander H. Pseudomonas aeruginosa bronchopulmonary infection in late human immunodeficiency $\mathbb{Q}$ virus disease. Am Rev Respir Dis 1993;148:992-6.

7 Graham N, Zeger SL, Lawrence PP, Vermund SH, Detels $\mathrm{R}$, Rinaldo CR, et al. The effects on survival of early $\mathrm{R}$, Rinaldo $\mathrm{CR}$, et al. The effects on survival of early Engl $\mathcal{F}$ Med 1992;326:1037-42.

8 Lemp GF, Hirozam AM, Cohen JB, Derish PA, McKiney KC, Hernandez SR. Survival for women and men with $\mathbb{D}$ AIDS. F Infect Dis 1992;166:74-9.

9 Doring G, Obernasser HJ, Botzchart K, Flehmig B, Hoiby N, Hofman A. Proteases of Pseudomonas aeruginosa in $\bigcirc$ patients with cystic fibrosis. F Infect Dis 1983;147:744-50. 응

10 Small CB, Kaufman ?, Armenaka M, Rosenstreich DL. Sinusitis and atopy in human immunodeficiency virus infection. $\mathcal{F}$ Infect Dis 1993;167:283-90.

11 Rubin JS, Honigberg R. Sinusitis in patients with the acquired immunodeficiency syndrome. ENT $f$ 1990;69: $460-3$.

12 Amman AJ, Schiffman G, Abrams D, Volberding P, Ziegler J, Conant M. B-cell immunodeficiency in acquired immune Conant $M$. B-cell immunodeficiency in acquired

deficiency syndrome. $\mathcal{Y} A M A$ 1984;251:1447-9.
13 Terpstra FG, Al BJ, Roos MT, DeWolf F, Goudsmit J, Schellekens PH, et al. Longitudinal study of leukocyte
PH function in homosexual men seroconverted for HIV: rapid and persistent loss of B cell function after HIV infection. Eur f Immunol 1989;19:667-73. 